\title{
Compact Stellar Systems in the Fornax Cluster: Super-massive Star Clusters or Extremely Compact Dwarf Galaxies?
}

\author{
M. J. Drinkwater ${ }^{1}$, J. B. Jones ${ }^{2}$, M. D. Gregg ${ }^{3}$ and S. Phillipps ${ }^{2}$ \\ ${ }^{1}$ School of Physics, University of Melbourne, Vic. 3010, Australia \\ m.drinkwater@physics.unimelb.edu.au \\ ${ }^{2}$ Department of Physics, University of Bristol, Tyndall Avenue, Bristol, BS8 1TL, UK \\ B.Jones, S.Phillipps@bristol.ac.uk \\ ${ }^{3}$ University of California, Davis, and Institute for Geophysics and Planetary Physics, \\ Lawrence Livermore National Laboratory, L-413, Livermore, CA 94550, USA \\ gregg@igpp.ucllnl.org
}

Received 1999 October 26, accepted 2000 March 30

\begin{abstract}
We describe a population of compact objects in the centre of the Fornax Cluster which were discovered as part of our 2dF Fornax Spectroscopic Survey. These objects have spectra typical of old stellar systems, but are unresolved on photographic sky survey plates. They have absolute magnitudes $-13<M_{B}<-11$, so they are 10 times more luminous than any Galactic globular clusters, but fainter than any known compact dwarf galaxies. These objects are all within 30 arcminutes of the central galaxy of the cluster, NGC 1399, but are distributed over larger radii than the globular cluster system of that galaxy. We suggest that these objects are either super-massive star clusters (intra-cluster globular clusters or tidally stripped nuclei of dwarf galaxies) or a new type of low-luminosity, compact elliptical dwarf ('M32-type') galaxy. The best way to test these hypotheses will be to obtain high-resolution imaging and high-dispersion spectroscopy to determine their structures and mass-to-light ratios. This will allow us to compare them to known compact objects and establish whether they represent a new class of hitherto unknown stellar system.
\end{abstract}

Keywords: galaxies: star clusters—galaxies: dwarf—galaxies: formation

\section{Introduction}

In cold dark matter (CDM) galaxy formation, small dense halos of dark matter collapse at high redshift and eventually merge to form the large virialised galaxy clusters seen today. The CDM model is very good at reproducing large-scale structure, but only very recently have the best numerical simulations (Moore et al. 1998) had the resolution to trace the formation of small halos within galaxy clusters, with masses $\approx 10^{9} \mathrm{M} \odot$. We do not yet know what the lower mass limit is for the formation of halos in the cluster environment; determining the lower limit of galaxy mass in clusters will provide an important constraint on these models. Most of the smallest cluster galaxies are low-surfacebrightness dwarfs, for which mass estimates are very difficult, although comparison with field low-surfacebrightness dwarfs would suggest that they may be darkmatter-dominated (e.g. Carignan \& Freeman 1988).

In this paper we describe a population of small objects we have found in the Fornax Cluster (see also Minniti et al. 1998; Hilker et al. 1999) which have high surface brightness. The origin and nature of these objects is not yet clear, but if they are a product of the galaxy formation process in clusters, their high surface brightness will make it possible to probe the low-mass limit discussed above. They may represent extreme examples of compact, low-luminosity ('M32-type') dwarf ellipticals. Alternatively, these objects may be super-massive star clusters-there is a very large population of globular clusters associated with the central galaxy of the Fornax Cluster, NGC 1399 (Grillmair et al. 1994). These objects are generally similar to Galactic globular clusters with similar colours and luminosities (Forbes et al. 1998). There is evidence that they are not all bound to the NGC 1399 system. Kissler-Patig et al. (1999) show that the kinematics of 74 of the globular clusters indicate that they are associated with the cluster gravitational potential, rather than that of NGC 1399. They infer that the most likely origin of these globular clusters is that they have been tidally stripped from neighbouring galaxies. This has also been suggested by West et al. (1995), although the effect would be diluted by the large number of halo stars that would presumably be stripped at the same time.

Bassino, Muzzio \& Rabolli (1994) suggest that the NGC 1399 globular clusters are remnants of the nuclei of dwarf nucleated galaxies that have survived the disrupture of being captured by the central cluster galaxy. A related suggestion from a second model proposed by West et al. (1995) is that intra-cluster globular clusters could have formed in situ in the cluster environment. Bassino et al. (1994) conclude their discussion by noting that remnant nuclei an order of magnitude larger (and more luminous) than standard globular clusters would also be formed in significant numbers, but that existing globular cluster searches would not have included them. In Section 2 of this paper, we describe how the observations of our Fornax Spectroscopic Survey have sampled this part of the cluster population by measuring optical spectra of all objects brighter than $B_{J}=19.7$ in the centre of the Fornax Cluster. In Section 3 we describe the properties of a new population of compact objects found in the cluster that appear to be inter- 
mediate in size between globular clusters and the smallest compact dwarf galaxies. We discuss the nature of these objects in Section 4, and show that higherresolution observations will enable us to determine if they are more like globular clusters or dwarf galaxies.

\section{Discovery Observations: The Fornax Spectroscopic Survey}

Our Fornax Spectroscopic Survey, carried out with the $2 \mathrm{dF}$ multi-object spectrograph on the Anglo-Australian Telescope (see Drinkwater et al. 2000), is now $87 \%$ complete in its first field to a limit of $B_{J}=19.7$. The $2 \mathrm{dF}$ field is a circle of diameter 2 degrees (i.e. $\pi$ square degrees of sky). We have measured optical spectra of some 4000 objects (some going fainter than our nominal limit) in a $2 \mathrm{dF}$ field centred on the central galaxy of the
Finally, in addition to the dwarf galaxies already listed in the Fornax Cluster Catalog (FCC, Ferguson 1989) which we have confirmed as cluster members, we have found a sample of five very compact objects at the cluster redshift which are unresolved on photographic sky survey plates and are not included in the FCC. These new members of the Fornax Cluster are listed in Table 1, along with their photometry measured from the UKST plates.

Our $2 \mathrm{dF}$ measurements of unresolved objects are $80 \%$ complete in the magnitude range of these objects $\left(17.5<b_{j}<20.0\right)$ There is therefore about one more similar compact object still to be found in our central $2 \mathrm{dF}$ field. The number density of these objects is therefore $6 \pm 3$ per $2 \mathrm{dF}$ field ( $\pi$ square degrees). Two of the objects (the two brightest) were also identified as cluster members by Hilker et al. (1999). Hilker et al. measured spectra of about 50 galaxies brighter than $V=$

Table 1. The new compact objects

\begin{tabular}{|c|c|c|c|c|c|c|}
\hline $\mathrm{N}$ & Name & RA (J2000) Dec & $\begin{array}{c}B_{J} \\
(\mathrm{mag})\end{array}$ & $\begin{array}{c}M_{B} \\
(\mathrm{mag})\end{array}$ & $\begin{array}{c}c z \\
\left(\mathrm{~km} \mathrm{~s}^{-1}\right)\end{array}$ & Notes \\
\hline$\overline{1}$ & FCSS J033703.3-353804 & $033703.30-353804.6$ & 19.85 & -11.1 & 1507 & \\
\hline 2 & FCSS J033806.3-352858 & $033806.33-352858.8$ & 18.85 & -12.1 & 1328 & \\
\hline 3 & FCSS J033854.1-353333 & $033854.10-353333.6$ & 17.68 & -13.2 & 1595 & 1 \\
\hline 4 & FCSS J033935.9-352824 & $033935.95-352824.5$ & 18.82 & -12.1 & 1936 & 2 \\
\hline 5 & FCSS J033952.5-350424 & $033952.58-350424.1$ & 19.66 & -11.2 & 1337 & \\
\hline
\end{tabular}

${ }^{1}$ CGF 1-4 ${ }^{2}$ CGF 5-4, both in Hilker et al. (1999)

Fornax Cluster (NGC 1399). This survey is unique in that the targets (selected from digitised UK Schmidt Telescope photographic sky survey plates) include all objects, both unresolved ('stars') and resolved ('galaxies') in this large area of sky. The resolved objects measured are mostly background galaxies, as expected, with a minor contribution from Fornax Cluster members. The unresolved objects are mostly Galactic stars and distant AGN, also as expected, but some are compact starburst (and post-starburst) galaxies beyond the Fornax Cluster (Drinkwater et al. 1999).
20 in a square region of width 0.25 degrees at the centre of the Fornax Cluster. In the 'galaxies' they include objects that were very compact, but still resolved. By contrast, our own survey covers a much larger area and also includes all unresolved objects.

\section{Properties of the Compact Objects}

3.1 Sizes

These object images are unresolved and are classified 'stellar' in our UKST plate data, although imaging with

\section{Object $1 \quad$ Object $2 \quad$ Object $3 \quad$ Object $4 \quad$ Object 5}

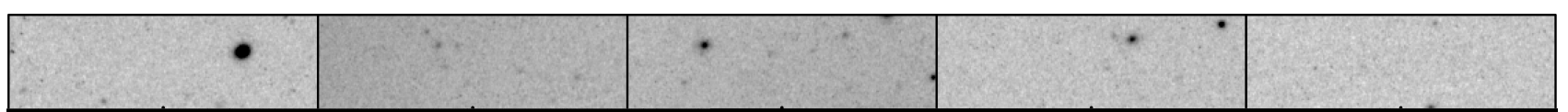

Figure 1-R-band photographic images of the new compact objects. The images are all from a single UKST exposure on Tech-Pan emulsion, digitised by SuperCOSMOS (Miller et al. 1992). Each image is 2.5 arcmin across, with North at the top and East to the left. 


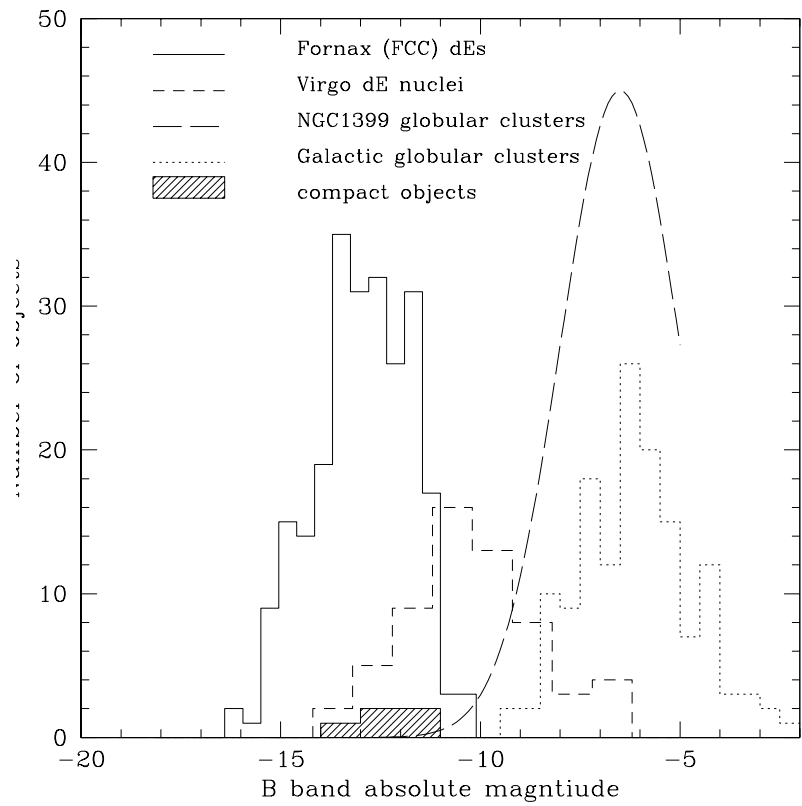

Figure 2-Absolute magnitude distribution of the compact objects (filled histogram) compared to those for dEs in the Fornax Cluster (Ferguson 1989; solid histogram), the nuclei of dE,Ns in the Virgo Cluster (Binggeli \& Cameron 1991; short dashes), a model fit to the globular clusters around NGC 1399 (Bridges, Hanes \& Harris 1991; long dashes) and Galactic globular clusters (Harris 1996; dotted). Note: The magnitude limit of our survey, in which the compact objects were found, corresponds to $M_{B}=-11$.

the CTIO Curtis Schmidt shows that the brightest two objects have marginal signs of extended structure. In Figure 1 we present R-band (Tech Pan emulsion + OG 590 filter) photographic images of these compact objects from the UKST. These were taken in seeing of about 2.2 arcsec FWHM and the third object (FCSS J033854.1353333 ) is resolved with a 3.2 arcsec FWHM. Applying a very simple deconvolution of the seeing, this corresponds to a scale size (HWHM) of about $80 \mathrm{pc}$. This is much larger than any known globular cluster, so this object, at least, is not a globular cluster. The other objects are all unresolved, so must have scale sizes smaller than this.

\subsection{Luminosity and Colours}

These new objects have absolute magnitudes $-13<M_{B}$ $<-11$, based on a distance modulus of 30.9 mag to the Fornax Cluster (Bureau, Mould \& Staveley-Smith 1996). These values are at the lower limit of dwarf galaxy luminosities (Mateo 1998), but the objects are much more luminous than any known Galactic globular cluster (Harris 1996) and the most luminous of the NGC 1399 globulars (Forbes et al. 1998), which have $M_{B} \approx-10$. The luminosities of the compact objects are compared to those for several other populations of dwarf galaxy and star clusters in Figure 2. We note that the magnitude limit of the $2 \mathrm{dF}$ data corresponds to an absolute magnitude of $M_{B} \approx-11$ here. In order of decreasing luminosity, the first comparison is with the dwarf ellipticals listed in the FCC as members of the
Fornax Cluster. The possible M32-type galaxies in the FCC are not included, as none of them have yet been shown to be cluster members (Drinkwater, Gregg \& Holman 1997). The figure shows that the Fornax dEs have considerable overlap in luminosity with the compact objects, but morphologically they are very different, being fully resolved, low-surface-brightness galaxies. Recently, several new compact dwarf galaxies have been discovered in the Fornax Cluster (Drinkwater \& Gregg 1998) but these are all brighter than $M_{B}=-14$ and do not match any of the objects we discuss here. Binggeli \& Cameron (1991) measured the luminosity function of the nuclei of nucleated dwarf elliptical galaxies in the Virgo Cluster. Figure 2 shows that this also overlaps the distribution of the new compact objects. In this case the morphology is the same, so the compact objects could originate from the dwarf nuclei. Figure 2 also shows the luminosity functions of both the NGC 1399 globular clusters (Bridges, Hanes \& Harris, 1991) and Galactic globular clusters (Harris 1996). These are quite similar and have no overlap with that for the new compact objects.

For completeness we note that the luminosities of the compact objects have considerable overlap with those of dwarf galaxies in the Local Group (Mateo 1998), but even the most compact of the Local Group dwarfs, Leo I $\left(M_{B}=-11.1\right)$ would be resolved $\left(r_{e} \approx 3^{\prime \prime}\right)$ in our images at the distance of Fornax. The only population they match in both luminosity and morphology is the bright end of the nuclei of nucleated dwarf ellipticals.

\subsection{Spectral Properties}

The $2 \mathrm{dF}$ discovery spectra of these compact objects are shown in Figure 3. They are similar to those of earlytype dwarf galaxies in the sample (two are shown for comparison in the Figure), with no detectable emission lines. As part of the spectral identification process in the Fornax Spectroscopic Survey, we cross-correlated all spectra with a sample of stellar templates from the Jacoby, Hunter \& Christian (1984) library. The spectra of the new compact objects were best fit by K-type stellar templates, consistent with an old (metal-rich) stellar population. The $\mathrm{dE}$ galaxies observed with the same system, by contrast, are best fit by younger $\mathrm{F}$ and early G-type templates. This gives some indication in favour of the compact objects being related to globular clusters, although we note that two of them were analysed by Hilker et al. (1999) in more detail without any conclusive results. We do not have the spectrum of a $\mathrm{dE}$ nucleus available for direct comparison, but since our $2 \mathrm{dF}$ spectra are taken through a 2 arcsec diameter fibre aperture, the spectrum of FCC 211, a nucleated dE, is dominated by the nucleus. This spectrum was fitted by a younger F-type stellar template, again suggestive of a younger population than the compact objects. We cannot draw any strong conclusions from these lowresolution, low-signal-to-noise spectra. 


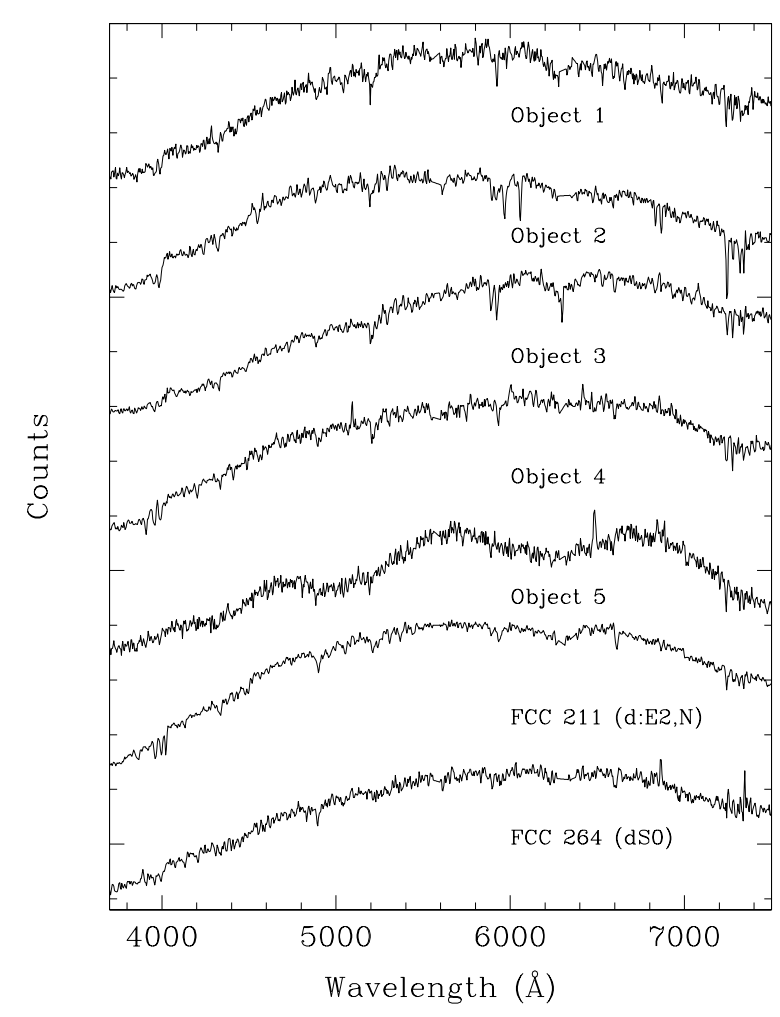

Figure 3-2dF discovery spectra of the five compact objects as well as two cluster dwarf galaxies for comparison. Note: the largescale ripple in the spectrum of the fifth object (FCSS J033952.5350424 ) is an instrumental effect caused by deterioration in the optical fibre used.

\subsection{Radial Distribution}

The main advantage of our survey over the previous studies of the NGC 1399 globular cluster system (e.g Grillmair et al. 1994; Hilker et al. 1999) is that we have complete spectroscopic data over a much larger field, extending to a radius of 1 degree (projected distance of $270 \mathrm{kpc}$ ) from the cluster centre. This means that we can determine the spatial distribution of the new compact objects. In Figure 4 we plot the normalised, cumulative radial distribution of the new compact objects, compared to that of foreground stars and cluster galaxies. This plot is the one used to calculate KolmogorovSmirnov (KS) statistics, and allows us to compare the distributions of objects independent of their mean surface densities. It is clear from Figure 4 that the new compact objects are very concentrated towards the centre of the cluster, at radii between 5 and 30 arcmin $(20-130 \mathrm{kpc})$. Their distribution is more centrally concentrated than the King profile fitted to cluster members by Ferguson (1989), with a core radius of 0.7 degrees $(190 \mathrm{kpc})$. The KS test gives a probability of 0.01 that the compact objects have the same distributions as the FCC galaxies; they are clearly not formed (or accreted) in the same way as average cluster galaxies. To test the hypothesis that the compact objects are formed from nucleated dwarfs, we also plot the distribution of all the FCC nucleated dwarfs, as these are more clustered than other dwarfs (Ferguson \& Binggeli 1994). However, in

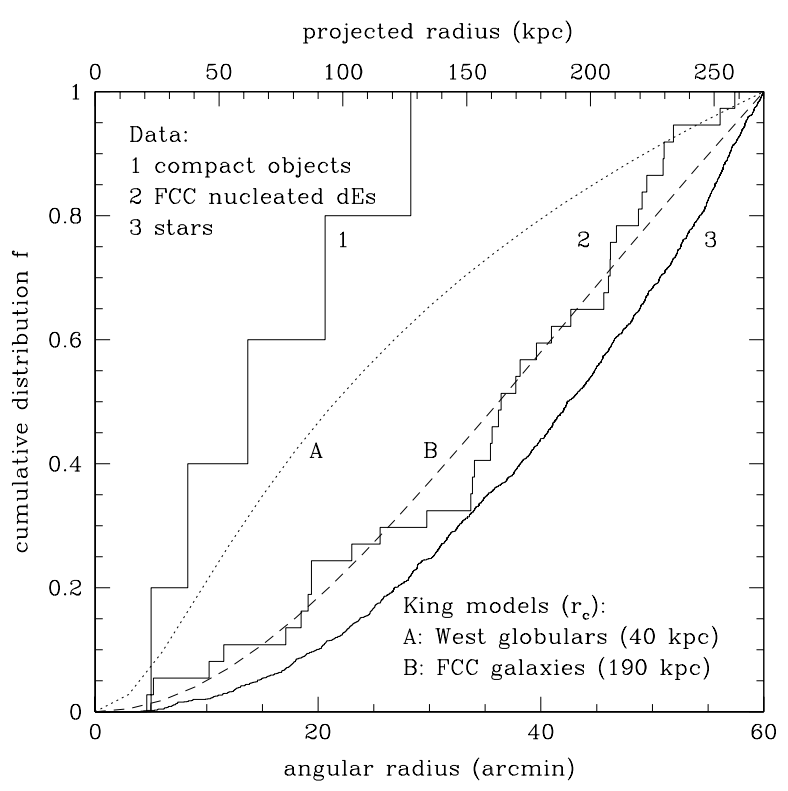

Figure 4-Cumulative radial distribution of the new compact objects compared to the predicted distribution for intra-cluster globular clusters (West et al. 1995) and the profile fit to the distribution of all FCC. Also shown is the distribution of all nucleated dwarfs in the FCC and all the unresolved objects (stars) observed in our $2 \mathrm{dF}$ survey.

the central region of interest here the nucleated dwarf profile lies very close to the King profile of all the FCC galaxies, so this does not provide any evidence for a direct link with the new compact objects.

West et al. (1995) suggest that a smaller core radius should be used for intra-cluster globular clusters (GCs). This profile, also shown in Figure 4, is more consistent with the distribution of the new objects: the KS probability of the compact objects being drawn from this distribution is 0.39 .

We also note that the radial distribution of the compact objects is much more extended than that of the NGC 1399 globular cluster system, as discussed by Grillmair et al., and extends to three times the projected radius of that sample. It is unlikely that all the compact objects are associated with NGC 1399. This is emphasised by a finding chart for the central 55 arcmin of the cluster, shown in Figure 5, which indicates the locations of the compact objects. They are widely distributed over this field, and the third object (FCSS J033854.1353333) in particular is much closer to NGC 1404, although we note that its velocity is not close to that of NGC 1404 (see below).

\subsection{Velocity Distribution}

We have some limited information from the radial velocities of the compact objects. The mean velocity of all five $\left(1530 \pm 110 \mathrm{~km} \mathrm{~s}^{-1}\right)$ is consistent with that of the whole cluster $\left(1540 \pm 50 \mathrm{~km} \mathrm{~s}^{-1}\right)$ (Jones \& Jones 1980). However, given the small sample, it is also consistent with the velocity of NGC $1399\left(1425 \pm 4 \mathrm{~km} \mathrm{~s}^{-1}\right)$, as might be expected for a system of globular clusters. 


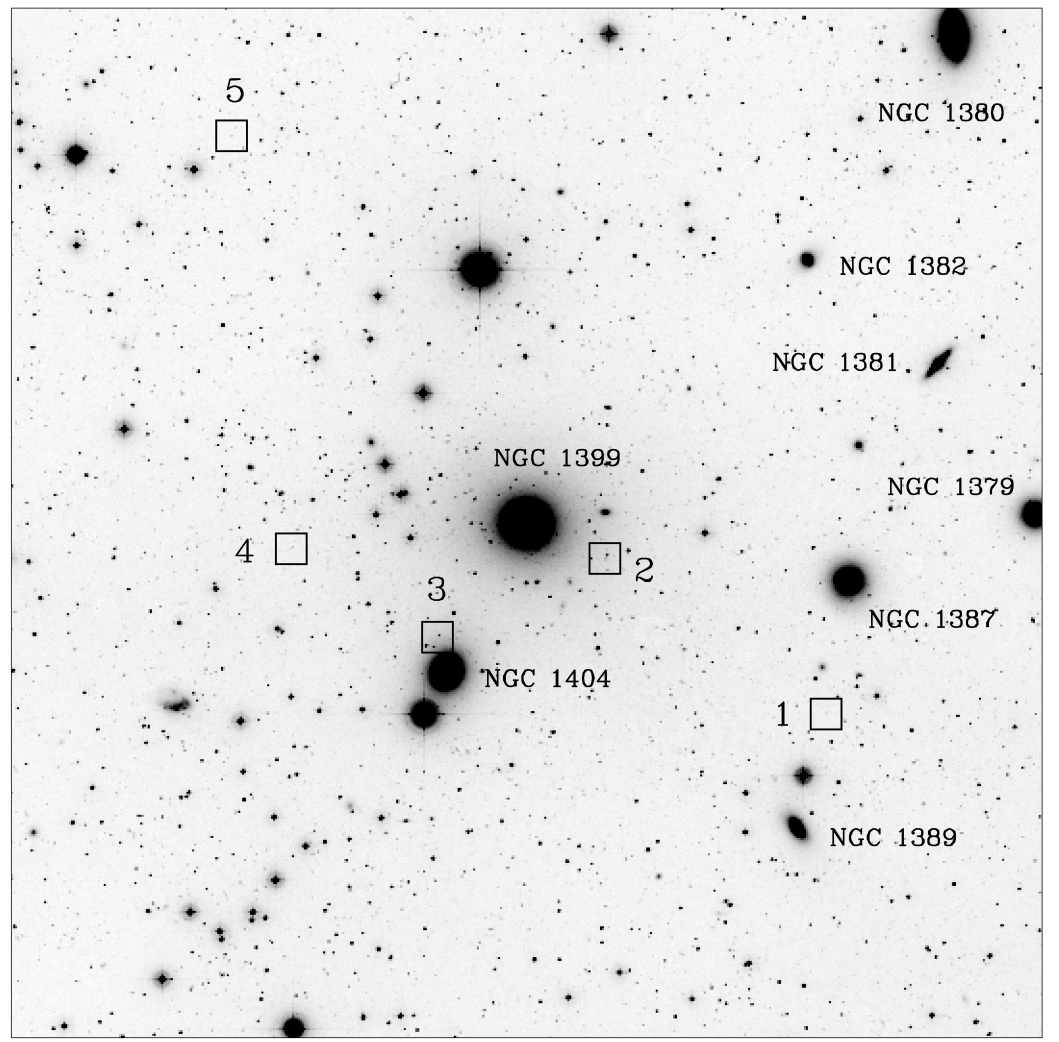

Figure 5-The central region of the Fornax Cluster, with the positions of the new compact objects indicated by squares. This R-band photographic image is from a single UKST exposure on Tech-Pan emulsion, digitised by SuperCOSMOS (Miller et al. 1992).

Interestingly, the analysis of the dynamics of 74 globular clusters associated with NGC 1399 by Kissler-Patig et al. (1999) notes that their radial velocity distribution has two peaks, at about 1300 and $1800 \mathrm{~km} \mathrm{~s}^{-1}$. Our sample is far too small to make any conclusions about the dynamics of these objects at present.

\section{Discussion}

We cannot say much more about the nature of these objects on the basis of our existing data. In groundbased imaging, they are intermediate between large GCs and small compact dwarf galaxies, so it becomes almost a matter of semantics to describe them as one or the other. The most promising way to distinguish between these possibilities is to measure their mass-tolight $(\mathrm{M} / \mathrm{L})$ ratios. If they are large, but otherwise normal, GCs, they will be composed entirely of stars, giving a very low $\mathrm{M} / \mathrm{L}$. If they are the stripped nuclei of dwarf galaxies we might expect them to be associated with some kind of dark halo, but we would not detect the dark halos at the small radii of these nuclei, so we would also measure small M/L values. Alternatively, these objects may represent a new, extreme class of compact dwarf elliptical ('M32-type') galaxy. These would presumably have formed through gravitational collapse within dark-matter halos, so would have high $\mathrm{M} / \mathrm{L}$ ratios, like dwarf galaxies in the Local Group (Mateo 1998). One argument against this interpretation is the apparent lack of M32-like galaxies at brighter luminosities (Drinkwater \& Gregg 1998). If the compact objects are dwarf galaxies, they will represent the faintest M32-like galaxies ever found. They may also fill in the gap between globular clusters and the fainter compact galaxies in the surface brightness vs magnitude distribution given by Ferguson \& Binggeli (1994).

A further possibility is that these are small-scalelength $(\sim 100 \mathrm{pc})$ dwarf spheroidal galaxies of only moderately low surface brightness. While Local Group $\mathrm{dSphs}$ of equivalent luminosity generally have substantially larger scale sizes (and consequently lower surface brightnesses) (Mateo 1998), Leo I for example has $M_{B}=-11.0$, and a scale length of only $110 \mathrm{pc}$ (Caldwell et al. 1992), but, as we discuss above, this would be resolved in our existing imaging.

Our existing data will only allow us to estimate a conservative upper limit to the masses of these objects. If we say that the core radii of the objects are less than $75 \mathrm{pc}$ and the velocity dispersions are less than 400 $\mathrm{km} \mathrm{s}^{-1}$ (the resolution of our $2 \mathrm{dF}$ spectra), we find that the virial mass must be less than $10^{10} \mathrm{M} \odot$. For a typical luminosity of $M_{B}=-12$, this implies that $\mathrm{M} / \mathrm{L}<2 \times 10^{3}$. This is not a very interesting limit, so we plan to reobserve these objects at higher spectral resolution from the ground and higher spatial resolution with the Hubble Space Telescope (HST) in order to be sensitive to $\mathrm{M} / \mathrm{L} \approx$ 


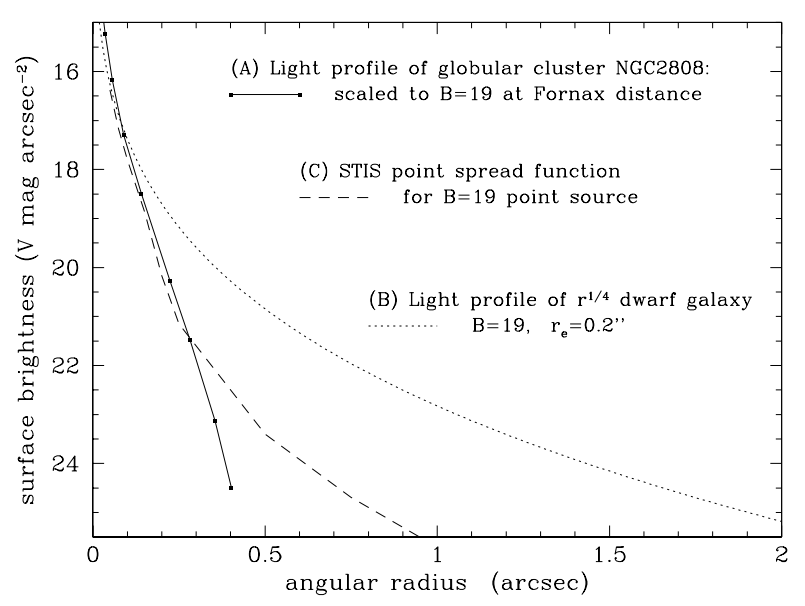

Figure 6-Predicted radial surface brightness profiles of the compact objects in two extreme cases: (A) a Galactic globular cluster (Illingworth \& Illingworth 1976) scaled 3 mag brighter in surface brightness, and (B) a compact dwarf galaxy with an $r^{1 / 4}$ profile. Both are scaled to have total magnitudes of $B=19 \mathrm{mag}$; they are not corrected for instrumental PSF, which is also shown (C).

100. This will allow us to distinguish globular clusters from dwarf galaxies.

In order to demonstrate what we could measure with high-resolution images, we present two extreme possibilities in Figure 6: a very compact Galactic globular cluster and a dwarf galaxy with an $r^{1 / 4}$ profile $\left(r_{e}=0.2\right.$ arcsec), both normalised to magnitudes of $B=19$ ( $V=$ 18.4 ) and the Fornax Cluster distance. We also plot the PSF of the Space Telescope Imaging Spectrograph (STIS) in the figure for reference. The globular cluster profile is that of NGC 2808 (Illingworth \& Illingworth 1976), with the radius scaled to the distance of the Fornax Cluster and the surface brightness then scaled to give the desired apparent magnitude. The globular cluster profile is very compact and will only just be resolved with HST, but it will clearly be differentiated from the dwarf galaxy profile.

In addition to measuring the sizes of these objects for the mass measurement, the radial surface brightness profiles may also give direct evidence for their origin and relationship to other kinds of stellar systems. For example, if they are the stripped nuclei of galaxies, the remnants of the outer envelope might show up in the HST images as an inflection in the surface brightness profile at large radius.

\section{Summary}

We have reviewed the observed properties of these new compact objects discovered in the Fornax Cluster. Their luminosities are intermediate between those of known globular clusters and compact dwarf galaxies, but they are consistent with the bright end of the luminosity function of the nuclei of nucleated dwarf ellipticals. The $2 \mathrm{dF}$ spectra are suggestive of old (metal-rich) stellar populations, more like globular clusters than dwarf galaxies. Finally, the radial distribution of the compact objects is more centrally concentrated than is usual for cluster galaxies in general, but extends further than that for the known globular cluster system of NGC 1399.

These objects are most likely either massive star clusters (extreme globular clusters or tidally-stripped dwarf galaxy nuclei) or very compact, low-luminosity dwarf galaxies. In the latter case these new compact objects would be very-low-luminosity counterparts to the peculiar compact galaxy M32. This would be particularly interesting, given the lack of M32-like galaxies at brighter luminosities (Drinkwater \& Gregg 1998). With higher resolution images and spectra, we will be able to measure the mass-to-light ratios of these objects and determine which of these alternatives is correct.

\section{Acknowledgments}

We thank the referee for helpful suggestions which have improved the presentation of this work. We wish to thank Dr Harry Ferguson for helpful discussions and for providing the STIS profile. This material is based on work supported by the National Science Foundation under grant no. 9970884. Part of the work reported here was done at the Institute of Geophysics and Planetary Physics, under the auspices of the US Department of Energy by Lawrence Livermore National Laboratory under contract no. W-7405-Eng-48. MJD acknowledges support from an Australian Research Council Large Grant.

\section{References}

Caldwell, N., Armandroff, T. E., Seitzer, P., \& Da Costa, G. S. 1992, AJ, 103, 840

Bassino, L. P., Muzzio, J. C., \& Rabolli, M. 1994, ApJ, 431, 634

Binggeli, B., \& Cameron, L. M. 1991, A\&A, 252, 27

Bridges, T. J., Hanes, D. A., \& Harris, W. E. 1991, AJ, 101, 469

Bureau, M., Mould, J. R., \& Staveley-Smith, L. 1996, ApJ, 463, 60

Carignan, C., \& Freeman, K. C. 1988, ApJ, 332, L33

Drinkwater, M. J., \& Gregg, M. D. 1998, MNRAS, 296, L15

Drinkwater, M. J., Gregg, M. D., \& Holman, B. A. 1997, in The Second Stromlo Symposium: The Nature of Elliptical Galaxies, ASP Conf. Ser. 116, ed. M. Arnaboldi, G. S. Da Costa \& P. Saha (San Francisco: ASP), p. 287

Drinkwater, M. J., Phillipps, S., Gregg, M. D., Parker, Q. A., Smith, R. M., Davies, J. I., Jones, J. B., \& Sadler, E. M. 1999, ApJ, 511, L97

Drinkwater, M. J., Phillipps, S., Jones, J. B., Gregg, M. D., Deady, J. H., Davies, J. I., Parker, Q. A., Sadler, E. M., \& Smith, R. M. 2000, A\&A, 355, 900

Ferguson, H. C. 1989, AJ, 98, 367

Ferguson, H. C., \& Binggeli, B. 1994, A\&AR, 6, 67

Forbes, D. A., Grillmair, C. J., Williger, G. M., Elson, R. A. W., \& Brodie, J. P. 1998, MNRAS, 293, 325

Grillmair, C. J., Freeman, K. C., Bicknell, G. V., Carter, D., Couch, W. J., Sommer-Larsen, J., \& Taylor, K. 1994, ApJ, 422, L9

Harris, W. E. 1996, AJ, 112, 1487

Hilker, M., Infante, L., Vieira, G., Kissler-Patig, M., \& Richtler, T. 1999, A\&AS, 134, 75

Illingworth, G., \& Illingworth, W. 1976, ApJS, 30, 227 
Jacoby, G. H., Hunter, D. A., \& Christian, C. A. 1984, ApJS, 56, 257

Jones, J. E., \& Jones, B. J. T. 1980, MNRAS, 191, 685

Kissler-Patig, M., Grillmair, C. J., Meylan, G., Brodie, J. P., Minniti, D., \& Goudfrooij, P. 1999, AJ, 117, 1206

Mateo, M. 1998, ARA\&A, 36, 435

Minniti, D., Kissler-Patig, M., Goudfrooij, P., \& Meylan, G. 1998, $\mathrm{AJ}, 115,121$
Miller, L. A., Cormack, W., Paterson, M., Beard, S., \& Lawrence, L. 1992, in Digitised Optical Sky Surveys, ed. H. T. MacGillivray \& E. B. Thomson (Dordrecht: Kluwer), p 133

Moore, B., Governato, F., Quinn, T., Stadel, J., \& Lake, G. 1998, ApJ, 499, L5

West, M. J., Cote, P., Jones, C., Forman, W., \& Marzke, R. O. 1995, ApJ, 453, L77 